\title{
Fluid simulations of non-resonant anisotropic ion heating
}

\author{
D. Laveder, T. Passot, and P. L. Sulem \\ Université de Nice-Sophia Antipolis, CNRS, UMR7293, Observatoire de la Côte d'Azur, BP 4229, 06304 Nice Cedex 4, \\ France
}

Correspondence to: P. L. Sulem (sulem@oca.eu)

Received: 15 March 2013 - Revised: 16 April 2013 - Accepted: 3 June 2013 - Published: 9 July 2013

\begin{abstract}
The finite Larmor radius (FLR)-Landau fluid model, which extends the usual anisotropic magnetohydrodynamics to magnetized collisionless plasmas by retaining linear Landau damping and finite Larmor radius corrections down to the sub-ionic scales in the quasi-transverse directions, is used to study the non-resonant heating of the plasma by randomly driven Alfvén waves. One-dimensional numerical simulations, free from any artificial dissipation, are used to analyze the influence on the thermal dynamics, of the beta parameter and of the separation between the driving and the ion scales. While the gyrotropic heat fluxes play a dominant role when the plasma is driven at large scales, leading to a parallel heating of the ions by Landau damping, a different regime develops when the driving acts at scales comparable to the ion Larmor radius. Perpendicular heating and parallel cooling of the ions are then observed, an effect that is mostly due to the work of the non-gyrotropic pressure force and that can be viewed as the fluid signature of the so-called stochastic heating. A partial characterization of the plasma by global quantities (such as the magnetic compressibility and the density-magnetic field correlations that provide information on the dominant type of waves) is also presented. The enhancement of the parallel electron heating by a higher level of fast magnetosonic waves is in particular pointed out.
\end{abstract}

Keywords. Interplanetary physics (solar wind plasma)

\section{Introduction}

It is now well established that, in the solar wind, the protons are subject to a significant heating in the direction perpendicular to the ambient magnetic field and are, in some regions, cooled in the parallel direction at a rate comparable to that of the perpendicular heating rate (Hellinger et al.,
2013, and references therein). A natural source of energy for this effect originates from waves and turbulence. Perpendicular heating can result from cyclotron resonance in particular near the solar corona, but such a process cannot be efficient in the regions of the solar wind where the dynamics at the ion scales can, as confirmed by recent measurements (Sahraoui et al., 2010; Roberts et al., 2013), be mostly described in terms of waves propagating in directions nearly perpendicular to the background magnetic field. The frequencies of these waves in the solar wind frame are much smaller than the proton gyrofrequency, and, in such a regime, the plasma heating is supposed to originate from the non-resonant action of low-frequency modes such as kinetic Alfvén waves (KAWs) (Wang et al., 2006; Chandran et al., 2010; Nariyuki et al., 2010, and reference therein). In this regime, the socalled stochastic heating resulting from particle acceleration due to electric field fluctuations at the scale of the ion Larmor radius indeed breaks the conservation of the magnetic moment and leads to an increase of the perpendicular temperature of the plasma (Wu and Yoon, 2007; Bourouaine et al., 2008). This effect was studied by considering the dynamics of particles propagating in the electromagnetic field associated with KAWs, in the absence of any feedback (Chandran et al., 2010). It was in particular noticed that, if an efficient action of a few individual Alfvén waves required unrealistically large amplitudes, a relatively weak level of Alfvénic fluctuations with a broad spectrum extending to the ion scale would provide an efficient effect ( $\mathrm{Lu}$ and Chen, 2010).

The fully nonlinear dynamics is captured by particles in cell (PIC) simulations, but such simulations are computationally demanding, as a huge number of quasi-particles per cell is to be retained in order to maintain a low numerical noise. It turns out that plasma heating can also be addressed using a fluid approach, provided it retains low-frequency kinetic effects (Landau damping and finite Larmor radius corrections) 
for scales comparable to the ion Larmor radius and below. Such a description is provided by the so-called finite Larmor radius (FLR)-Landau fluid model (Passot and Sulem, 2007; Passot et al., 2012) that, within a self-consistent approach, can reproduce both heating and cooling processes, depending on the plasma parameters. As a first step, the problem is here addressed in one space dimension with the numerical code previously used to simulate the development of mirror modes and their feedback action on the temperatureanisotropy growth, which constrains the system to remain close to the instability threshold (Laveder et al., 2011). This one-dimensional setting enables us to address in detail the basic mechanisms at the origin of the non-resonant heating processes due to the waves and to their interaction with the particles, while the heating by current sheets and reconnection events (Karimabadi et al., 2013), also present in a turbulent flow, requires three-dimensional simulations.

The paper is organized as follows. Section 2 provides a brief description of the FLR-Landau fluid model. Section 3 specifies the conditions of the simulations together with a convenient framework to estimate plasma heating. Section 4 describes the thermal dynamics of the ions in both parallel and perpendicular directions when the beta parameter and the separation between the driving and ion scales are varied. Section 5 analyzes the relative importance of the various heating mechanisms, depending on the parameters. Characterization of the plasma by global quantities sensitive to the various kinds of waves is presented in Sect. 6 where the influence of the level of fast magnetosonic waves on the (parallel) electron heating is also discussed. Section 7 provides a few concluding remarks.

\section{The FLR-Landau fluid model}

It is well known that the magnetohydrodynamic (MHD) and bifluid descriptions are poorly adapted to the solar wind due to its collisionless character. These models in particular overestimate the compressibility, and, as recently shown in $\mathrm{Hu}-$ nana et al. (2011), Passot et al. (2012), and Hunana et al. (2013), pressure anisotropy and Landau resonance need to be retained even at large scales. Furthermore, permitting the development of anisotropic pressure may lead to the onset of micro-instabilities that are arrested at small scales by finite Larmor radius (FLR) contributions. In order to cope with this situation, the FLR-Landau fluid model was developed (Passot and Sulem, 2007; Passot et al., 2012), which extends the anisotropic MHD into a fluid description that accurately reproduces the linear kinetic theory, provided no ion-cyclotron resonance is encountered. It in particular captures the dynamics of quasi-transverse sub-ionic modes that are characterized by low frequencies compared with the gyrofrequency. This approach thus appears suitable for a magnetized plasma where the presence of the guide field prescribes a strong anisotropy of the energy transfer.
The FLR-Landau fluid model is constructed by deriving from the Vlasov-Maxwell system a moment hierarchy that starts with equations for the plasma density $\rho$ and for the ion velocity $u$ where, for the sake of simplicity, the electric field is expressed by the generalized Ohm's law, obtained after neglecting electron inertia. The ion pressure tensor includes both gyrotropic and non-gyrotropic contributions $\mathbf{p}_{\mathrm{i}}=p_{\perp \mathrm{i}} \mathbf{I}+\left(p_{\| \mathrm{i}}-p_{\perp \mathrm{i}}\right) \boldsymbol{\tau}+\Pi_{\mathrm{i}}$ with $\boldsymbol{\tau}=\widehat{b} \otimes \widehat{b}(\widehat{b}=b /|b|$ is the unit vector the direction of the local magnetic field). Differently, as we do not address the dynamics at the electron scale, the non-gyrotropic contribution is neglected in the electron pressure tensor $\mathbf{p}_{\mathrm{e}}=p_{\perp \mathrm{e}} \mathbf{I}+\left(p_{\| \mathrm{e}}-p_{\perp \mathrm{e}}\right) \widehat{b} \otimes \widehat{b}$. The equations governing the gyrotropic pressures and heat fluxes $q_{\perp r}$ and $q_{\| r}$ of each particle species $r$ are given in Passot and Sulem (2007) and Passot et al. (2012). The model involves the gyrotropic fourth-rank velocity cumulants and also the non-gyrotropic contributions to all the retained moments, which are estimated in a way that fits the low-frequency linear kinetic theory near an equilibrium state characterized by the instantaneous mean pressures and temperatures of the plasma. Explicit expressions of the various contributions in the present setup where the space variations only take place in a direction making a prescribed angle with the ambient magnetic field are given in Borgogno et al. (2007) and Camporeale et al. (2010). The lack of space does not enable us to provide a comprehensive description of the model. We nevertheless briefly mention that the starting point to close the hierarchy and to estimate the non-gyrotropic contributions consists in deriving the low-frequency kinetic expressions of the various moments that typically depend on electromagnetic field components and involve the plasma dispersion function (which is nonlocal both in space and time). These various expressions are then expressed in terms of other fluid moments in such a way as to minimize the occurrence of the plasma dispersion function (which is nonlocal in time and thus not suitable for addressing initial value problems). The latter is otherwise replaced by suitable Padé approximants, thus leading to local-in-time expressions involving in some places a Hilbert transform with respect to the longitudinal space coordinate, which appears as the signature of Landau damping within a fluid description.

\section{Measuring the plasma heating}

In order to mimic the energy injection near the ion gyroscale, due to the solar-wind Alfvén-wave cascade, and to study the resulting heating of the plasma, the FLR-Landau-fluid equations are supplemented by a random driving acting on the velocity component $u_{\mathrm{y}}$ perpendicular to the plane defined by the ambient magnetic field and the direction of propagation. This forcing is centered about a scale $l_{\mathrm{f}}$ with Fourier coefficients of fixed amplitudes and random phases chosen independently at each time step. The driving is only active when the energy per unit length of the perpendicular kinetic 
and magnetic fluctuations (which are mostly associated with Alfvén waves) $E_{\mathrm{M}}=\frac{1}{2}\left\langle\rho u_{\mathrm{y}}^{2}\right\rangle+\frac{1}{2}\left\langle b_{\mathrm{y}}^{2}\right\rangle$ remains below a fixed threshold, chosen to ensure a prescribed fluctuation level. Driving the velocity field only has the advantage of not directly affecting the space-averaged magnetic moment per unit mass $\mu_{\perp}=\left\langle\frac{p_{\perp \mathrm{i}}}{|b|}\right\rangle$ that obeys

$$
\begin{aligned}
& \frac{d \mu_{\perp}}{d t}=\left\langle\frac{p_{\perp \mathrm{i}}}{|b|^{2}} \widehat{b} \cdot\left[\nabla \times \frac{m_{\mathrm{i}} c}{\rho e}\left(\frac{j \times b}{c}-\nabla \cdot \mathbf{p}_{\mathrm{e}}\right)\right]\right\rangle \\
& +\left\langle\frac{1}{2|b|} \nabla \cdot S_{\perp \mathrm{i}}^{\perp}\right\rangle \\
& -\left\langle\frac{1}{2|b|}\left[\operatorname{tr}\left(\boldsymbol{\Pi}_{\mathrm{i}} \cdot \nabla u_{\mathrm{i}}\right)^{s} \quad-\left(\boldsymbol{\Pi}_{\mathrm{i}} \cdot \nabla u_{\mathrm{i}}\right)^{s}: \boldsymbol{\tau}+\boldsymbol{\Pi}_{\mathrm{i}}: \frac{d \boldsymbol{\tau}}{d t}\right]\right\rangle .
\end{aligned}
$$

In the parallel direction, we similarly define $\mu_{\|}=\left\langle\frac{|b|^{2} p_{\| \mathrm{i}}}{\rho^{2}}\right\rangle$ that obeys

$$
\begin{aligned}
\frac{d \mu_{\|}}{d t}= & -\left\langle\frac{2 p_{\| \mathrm{i}}}{\rho^{2}} \widehat{b} \cdot\left[\nabla \times \frac{m_{\mathrm{i}} c}{\rho e}\left(\frac{j \times b}{c}-\nabla \cdot \mathbf{p}_{\mathrm{e}}\right)\right]\right\rangle \\
& -\left\langle\frac{|b|^{3}}{\rho^{2}}\left[\nabla \cdot\left(q_{\| \mathrm{i}} \widehat{b}\right)-2 q_{\perp \mathrm{i}} \nabla \cdot \widehat{b}\right]\right\rangle-\left\langle\frac{|b|^{2}}{\rho^{2}} \nabla \cdot S_{\perp \mathrm{i}}^{\|}\right\rangle \\
& -\left\langle\frac{|b|^{2}}{\rho^{2}}\left[\left(\boldsymbol{\Pi}_{\mathrm{i}} \cdot \nabla u_{\mathrm{i}}\right)^{s}: \boldsymbol{\tau}-\boldsymbol{\Pi}_{\mathrm{i}}: \frac{d \boldsymbol{\tau}}{d t}\right]\right\rangle .
\end{aligned}
$$

Note that $\mu_{\perp}$ and $\mu_{\|}$differ from the space-averaged adiabatic invariants of the CGL theory (Chew et al., 1956) by a $\rho$ factor in the denominator.

In the right-hand side (r.h.s.) of the above equations, the first term originates from the Hall term (including the electron pressure gradient). The other contributions involve the gyrotropic $\left(q_{\| \mathrm{i}}\right.$ and $\left.q_{\perp \mathrm{i}}\right)$ and non-gyrotropic components $\left(S_{\perp \mathrm{i}}^{\|}\right.$ and $S_{\perp \mathrm{i}}^{\perp}$ ) of the parallel and perpendicular heat flux vectors, and the non-gyrotropic pressure $\Pi_{\mathrm{i}}$ (see Passot and Sulem, 2007, or Passot et al., 2012, for precise definitions of the notations). Since the evolution of $\mu_{\perp}$ is only due to the Hall effect and to the non-gyrotropic pressures and heat fluxes, this quantity is conserved in the limit where the system is driven at scales much larger than the ion scales. Differently, the evolution of $\mu_{\|}$is sensitive to the parallel and perpendicular gyrotropic fluxes that remain relevant in the large-scale limit.

As seen in the following, the usefulness of $\mu_{\perp}$ and $\mu_{\|}$is due to the fact that their time evolutions are very similar to those of the space-averaged perpendicular and parallel ion temperatures $\bar{T}_{\perp \mathrm{i}}$ and $\bar{T}_{\| \mathrm{i}}$, which obey ( $n$ denotes the number density)

$$
\begin{aligned}
& \frac{d}{d t} \bar{T}_{\perp \mathrm{i}}=\left\langle T_{\perp \mathrm{i}} \widehat{b} \cdot \nabla u \cdot \widehat{b}\right\rangle \\
& -\left\langle\frac{1}{n}\left[2 q_{\perp \mathrm{i}}(\nabla \cdot \widehat{b})+(\widehat{b} \cdot \nabla) q_{\perp \mathrm{i}}-q_{\| \mathrm{i}} \widehat{b} \cdot \nabla \widehat{b} \cdot \widehat{b}\right]\right\rangle \\
& -\left\langle\frac{1}{n}\left[\nabla \cdot S_{\perp \mathrm{i}}^{\perp}-\widehat{b} \cdot \nabla \widehat{b} \cdot S_{\perp \mathrm{i}}^{\|} \mathrm{]}\right\rangle\right. \\
& -\frac{1}{2}\left\langle\frac{1}{n}\left[\left(\operatorname{tr}(\Pi \cdot \nabla u)^{S}-(\Pi \cdot \nabla u)^{S}: \boldsymbol{\tau}+\Pi: \frac{d \boldsymbol{\tau}}{d t}\right)\right]\right\rangle, \\
& \frac{d}{d t} \bar{T}_{\| \mathrm{i}}=\left\langle\bar{T}_{\| \mathrm{i}} \nabla \cdot u\right\rangle-2\left\langle\bar{T}{ }_{\| \mathrm{i}} \widehat{b} \cdot \nabla u \cdot \widehat{b}\right\rangle \\
& +\left\langle\frac{1}{n}\left[2 q_{\| \mathrm{i}} \widehat{b} \cdot \nabla \widehat{b} \cdot \widehat{b}\right]\right\rangle-\left\langle\frac{1}{n}\left[\nabla \cdot S_{\perp p}^{\|}-2 \widehat{b} \cdot \nabla \widehat{b} \cdot S_{\perp \mathrm{i}}^{\|}\right]\right\rangle \\
& -\left\langle\frac{1}{n}\left[(\Pi \cdot \nabla u)^{S}: \boldsymbol{\tau}-\Pi: \frac{d \boldsymbol{\tau}}{d t}\right]\right\rangle .
\end{aligned}
$$

It is noticeable that the equations for $\mu_{\perp}$ and $\mu_{\|}$involve the Hall term, while the equations for the temperatures include mechanical terms associated with the work of the gyrotropic pressure force. By simple inspection, there is no clear evidence that $\bar{T}_{\perp \mathrm{i}}$ remains constant when the dynamics is concentrated at large scales. Considering $\mu_{\perp}$ and $\mu_{\|}$as a surrogate of the temperatures thus offers a better insight into the physical processes relevant for the heating of the plasma.

Note that, in the above temperature equations and also to leading order in the $\mu$ equations, the non-gyrotropic pressure and Hall contributions that arise in equations for the parallel quantities also occur with an opposite sign in the equations for the perpendicular ones. The latter also include a contribution involving $\operatorname{tr}(\Pi \cdot \nabla u)^{S}$ that turns out to be always positive in our simulations and thus heats the plasma.

In the numerical code, the FLR-Landau fluid equations are written using units based on the magnitude $B_{0}$ of the ambient field, the Alfvén velocity $v_{\mathrm{A}}$, the ion inertial length $d_{\mathrm{i}}=v_{\mathrm{A}} / \Omega$ (where $\Omega$ is the ion gyrofrequency), together with the uniform initial ion density $\rho_{0}$ and parallel pressure $p_{\| \text {i } 0}$. Integration is performed using a spectral method with an effective resolution of 256 grid points (after a partial dealiasing where the nonlinear terms are evaluated with twice the number of Fourier modes), in a periodic domain along a direction making an angle of $80^{\circ}$ with the ambient magnetic field. The injection scale $l_{\mathrm{f}}$ is associated with the Fourier mode of index 4 . Changing $l_{\mathrm{f}}$ is thus performed by changing the size of the integration domain. The driving term is turned on and off in order to maintain the transverse energy per unit length $E_{\mathrm{M}}$ close to 0.016 , leading to a root mean square amplitude of the transverse magnetic field and velocity fluctuations of about $12 \%$. The initial ion and electron temperatures are taken isotropic and equal. The time discretization is made using a third-order Runge-Kutta scheme with a time step $\Delta t$ ranging between $5 \times 10^{-5}$ and $2 \times 10^{-3}$ (in $\Omega^{-1}$ units). We explored different regimes by varying the ion parallel beta $\beta=8 \pi p_{\| \mathrm{i} 0} / B_{0}^{2}$ and the ratio between the driving scale $l_{\mathrm{f}}$ and either the ion inertial length $d_{\mathrm{i}}$ or the ion Larmor radius 


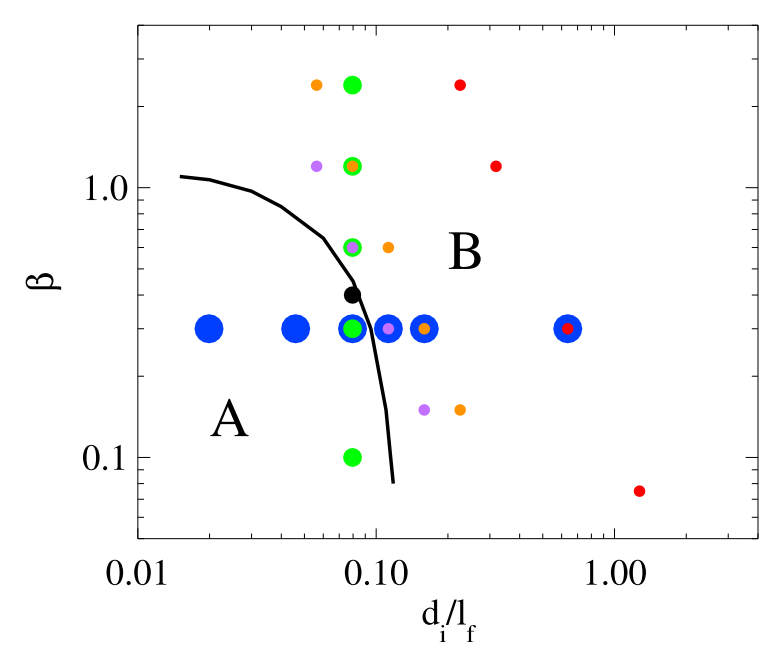

Fig. 1. Parameter plane $\left(d_{\mathrm{i}} / l_{\mathrm{f}}, \beta\right)$ where the various colors refer to the series of runs discussed in the text. This plane is divided into two regions displaying a different heating dynamics: in region $\mathrm{A}$, ion parallel heating dominates, while in region $\mathrm{B}$, perpendicular heating and parallel cooling take place.

$r_{\mathrm{L}}=d_{\mathrm{i}} \sqrt{\beta}$. Note that, in the case of one-dimensional simulations, the injection scale has a deep influence on the thermal dynamics, as the energy hardly cascades to small scales. Figure 1 provides an overview of the selected simulations in the parameter plane $\left(d_{\mathrm{i}} / l_{\mathrm{f}}, \beta\right)$, which turns out to be divided into two regions where the ion heating displays significantly different properties. The various colors refer to specific series of runs discussed in the following. All the simulations were performed without any artificial dissipation.

\section{Evolution of the temperatures}

Figure 2 displays the time evolution of the mean temperatures $\bar{T}_{\| \mathrm{i}}$ and $\bar{T}_{\perp \mathrm{i}}$, and of the corresponding $\mu_{\|}$and $\mu_{\perp}$, in the case of simulations performed with $\beta=0.3$ but decreasing driving scales $d_{\mathrm{i}} / l_{\mathrm{f}}=0.020$ (left panel), 0.080 (middle panel) and 0.16 (right panel). These runs belong to the series of simulations indicated by blue points in Fig. 1 and display three typical thermal behaviors of the plasma: in the left panel, $\bar{T}_{\| \mathrm{i}}$ increases while $\bar{T}_{\perp \mathrm{i}}$ stays almost constant; in the middle panel, both temperatures increase possibly at different rates that vary in time; in the right panel, $\bar{T}_{\perp \mathrm{i}}$ increases while $\bar{T}_{\| \mathrm{i}}$ decreases, leading eventually to the development of mirror instability.

As previously announced, in all the simulations the mean temperatures and the corresponding $\mu$ values display the same global behavior (an effect analyzed in Appendix A), up to a possible shift originating from a brief early-time temperature oscillation, especially in the case of a large-scale driving. Furthermore, and in particular in the latter regime, the temperatures display oscillations with a frequency matching the Alfvén wave frequency at the driving scale $l_{\mathrm{f}}$, which are smoothed out at the level of the $\mu$ 's. When measuring the different terms in the r.h.s. of Eqs. (3)-(4), it turns out that these oscillations are mostly due to the term proportional to $\widehat{b} \cdot \nabla u \cdot \widehat{b}$, and in particular to its leading contribution $\widehat{b}_{\mathrm{z}}\left(\partial_{\mathrm{z}} u_{\mathrm{y}}\right) \widehat{b}_{\mathrm{y}}$, which involves the driven velocity component $u_{\mathrm{y}}$. These oscillations are however not relevant for the plasma heating, on long time scales. In the following, we thus concentrate on the $\mu$ 's that display simpler properties.

The evolution of $\mu_{\perp}$ results only from small-scale contributions, namely the Hall term, the non-gyrotropic pressure tensor (often referred to as FLRs for shortness in the following) and also the non-gyrotropic heat fluxes, which turn out to be always subdominant and will thus be ignored in the following discussions. As a consequence, in simulations where injection takes place at large enough scales (a regime similar to that of Fig. 2, left), $\mu_{\perp}$ is conserved. Differently, when the driving acts at smaller scales, the Hall term and FLR contributions break the conservation of the perpendicular moment (middle and right panels), which then increases in time. The evolution of $\mu_{\|}$is in contrast sensitive to the parallel and perpendicular gyrotropic heat fluxes that remain significant at large scales and lead to a parallel heating of the ions by Landau damping (Fig. 2, left and middle). Note that in the right panel of Fig. 2, the enhancement of the heating and cooling processes shortly before the plasma becomes mirror unstable, which, in the present simulation, occurs at $t \approx 1200$. Furthermore, the thermal dynamics is significantly slower when the scale separation is larger.

Another important parameter that affects the ion heating is $\beta$. When considering the influence of this parameter for a fixed scale separation, it is important to specify whether the prescribed quantity is the ratio of the injection scale to the ion inertial length or to the ion Larmor radius. In the former case $\left(d_{\mathrm{i}} / l_{\mathrm{f}}=0.080\right)$, Fig. 3 displays, for $\beta=0.1$ (black), $\beta=0.3$ (blue), $\beta=0.6$ (green), and $\beta=2.4$ (red), the variation of $\mu_{\perp}$ (top panel) and $\mu_{\|}$(bottom panel). These simulations belong to the series indicated by green points in Fig. 1. At $\beta=0.1$, the perpendicular heating is very weak. It is significantly enhanced when $\beta$ is increased, while the parallel heating rate decreases and even becomes negative at large enough $\beta$. It follows that increasing $\beta$ while keeping constant the ratio $d_{\mathrm{i}} / l_{\mathrm{f}}$ is qualitatively similar to decreasing the driving scale at fixed $\beta$.

Since the evolution of the $\mu$ 's is influenced by nongyrotropic contributions for which the relevant scale is the ion Larmor radius, we consider the evolution of $\mu_{\|}$and $\mu_{\perp}$ at fixed $r_{\mathrm{L}} / l_{\mathrm{f}}$, with the series of runs indicated by red $\left(r_{\mathrm{L}} / l_{\mathrm{f}}=0.35\right)$, orange $\left(r_{\mathrm{L}} / l_{\mathrm{f}}=0.080\right)$ and violet $\left(r_{\mathrm{L}} / l_{\mathrm{f}}=\right.$ 0.062 ) points in Fig. 1. Figure 4 displays the time variation of $\mu_{\|}$and $\mu_{\perp}$ for $r_{\mathrm{L}} / l_{\mathrm{f}}=0.35$ (top panel) and $r_{\mathrm{L}} / l_{\mathrm{f}}=0.080$ (bottom panel), when $\beta$ takes the values 0.075 (black), 0.15 (magenta), 0.3 (blue), 1.2 (green) and 2.4 (red). The parallel quantities are indicated by dashed lines and the perpendicular ones by solid lines. In all these simulations, we observe perpendicular heating and parallel cooling, which eventually 

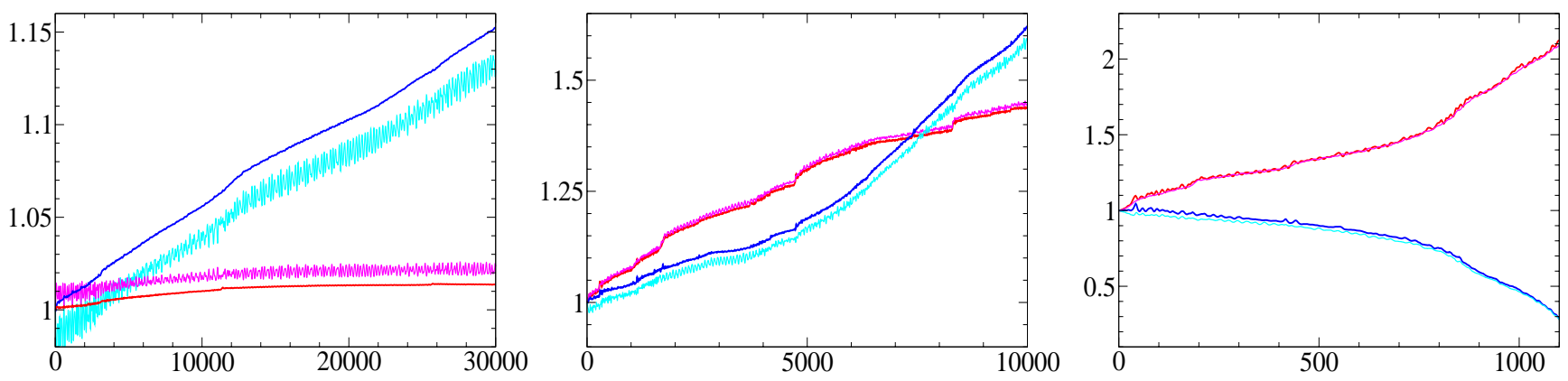

Fig. 2. Time variation of $\mu_{\perp}$ (red) and $\bar{T}_{\perp}$ (magenta), and also of $\mu_{\|}$(blue) and $\bar{T}_{\|}$(cyan), at fixed $\beta=0.3$ and three scale separations (from the blue series of Fig. 1) $d_{\mathrm{i}} / l_{\mathrm{f}}=0.020$ (left), $d_{\mathrm{i}} / l_{\mathrm{f}}=0.080$ (middle), and $d_{\mathrm{i}} / l_{\mathrm{f}}=0.16$ (right).

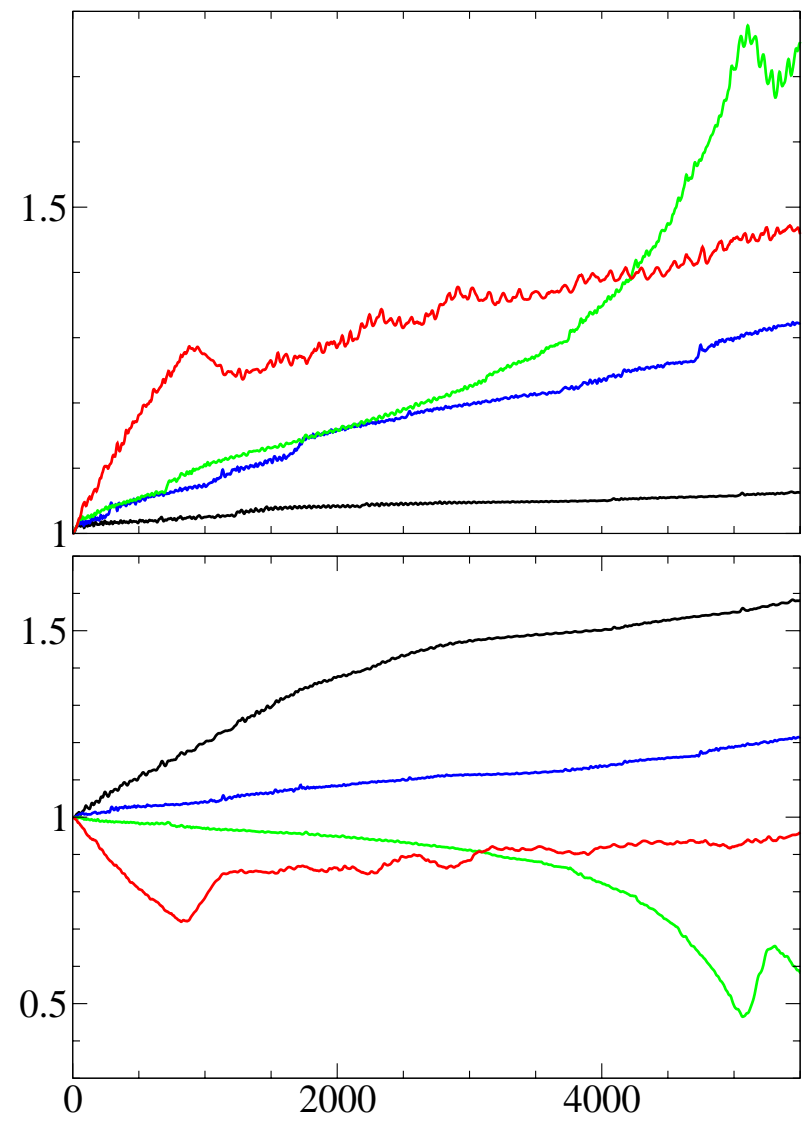

Fig. 3. Time variation of $\mu_{\perp}$ (top panel) and $\mu_{\|}$(bottom panel), for a fixed ratio $d_{\mathrm{i}} / l_{\mathrm{f}}=0.080$ and (green series of Fig. 1) $\beta=0.1$ (black), $\beta=0.3$ (blue), $\beta=0.6$ (green), and $\beta=2.4$ (red).

leads to mirror instability. Especially in the top panel corresponding to the smaller injection scale, the parallel cooling is rather weak and it does not vary significantly from one run to the other, while the efficiency of the perpendicular heating decreases when increasing $\beta$. A similar variation is observed in the particle simulations reported in Chandran et al. (2010). This contrasts with the simulations characterized by a fixed ratio $d_{\mathrm{i}} / l_{\mathrm{f}}$, for which the effect of changing $\beta$ is just the oppo-
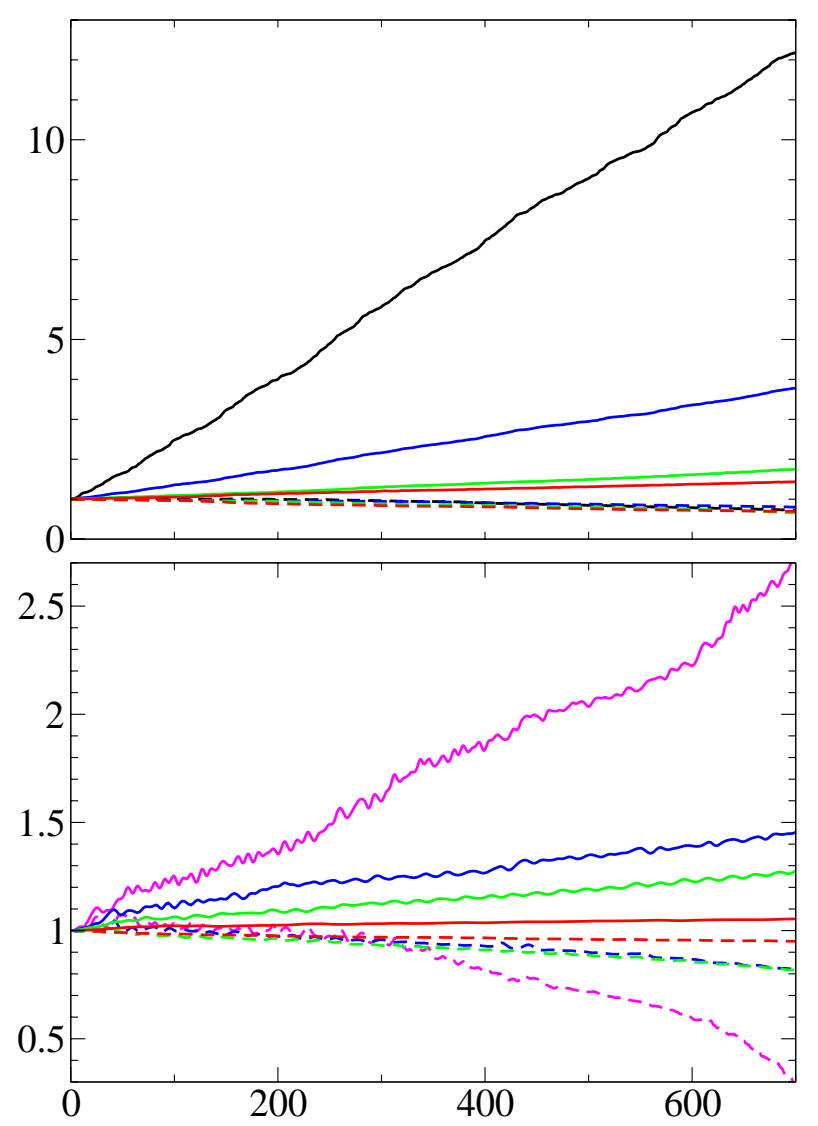

Fig. 4. Time variation of $\mu_{\perp}$ (solid lines) and $\mu_{\|}$(dashed lines), when fixing $r_{\mathrm{L}} / l_{\mathrm{f}}$ and changing $\beta$. Top: $r_{\mathrm{L}} / l_{\mathrm{f}}=0.35$ (red series of Fig. 1) with $\beta=0.075$ (black), $\beta=0.3$ (blue), $\beta=1.2$ (green), and $\beta=2.4$ (red). Bottom: $r_{\mathrm{L}} / l_{\mathrm{f}}=0.087$ (orange series of Fig. 1) with $\beta=0.15$ (magenta), $\beta=0.3$ (blue), $\beta=1.2$ (green), and $\beta=2.4$ (red).

site. We observe in the bottom panel a conspicuous enhancement of the heating when approaching the mirror threshold (for the run corresponding to the magenta curve, the mirror instability occurs at $t \approx 730$ ). 

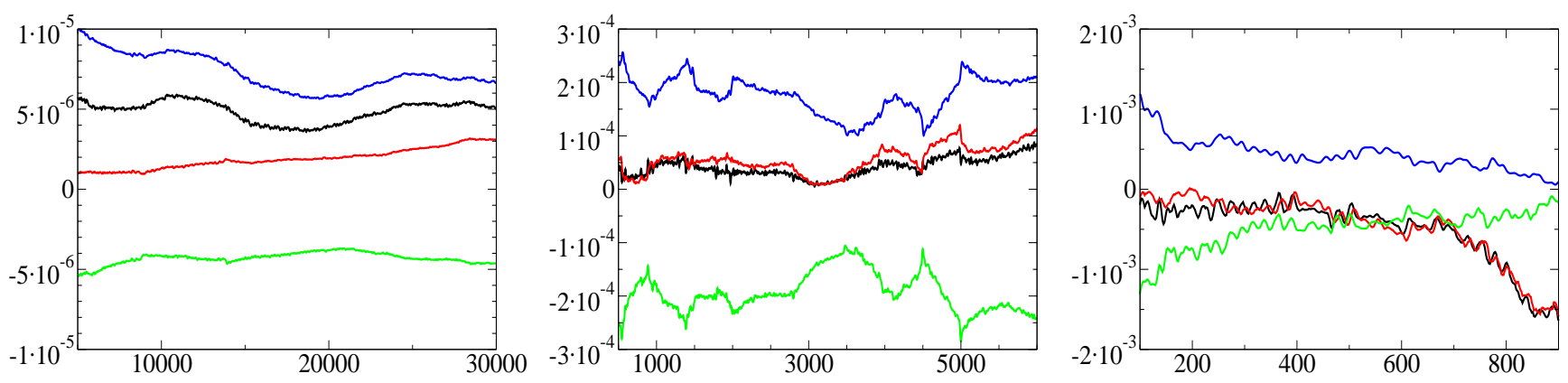

Fig. 5. Time evolution of the r.h.s. (black) of the equation for $\mu_{\|}$together with the various contributions: gyrotropic heat fluxes (blue), Hall effect (green), non-gyrotropic pressure (red), for the same simulations as in Fig. $2: \beta=0.3$ and $d_{\mathrm{i}} / l_{\mathrm{f}}=0.020\left(\right.$ left), $d_{\mathrm{i}} / l_{\mathrm{f}}=0.080$ (middle), and $d_{\mathrm{i}} / l_{\mathrm{f}}=0.16$ (right)

To summarize, the $\left(d_{\mathrm{i}} / l_{\mathrm{f}}, \beta\right)$ plane displayed in Fig. 1 can be divided into two regions. Although a sharp boundary can hardly be specified, an approximate separatrix can empirically be defined. The region denoted by " $\mathrm{A}$ " is characterized by a parallel ion heating and a weak to moderate perpendicular one, while in region B the plasma is subject to a strong perpendicular heating and to a parallel cooling, leading to mirror instability. Close to this separatrix, the system may hesitate between these two possible outcomes, and runs characterized by identical initial conditions and a driving corresponding to different realizations of the same random forcing can display different global evolutions.

\section{The heating mechanisms}

\subsection{The transition from parallel heating to cooling}

The dynamics is simple when the forcing is applied at very large scales (especially at low $\beta$ ), as in this case only the heat fluxes are relevant and the only measurable effect is a growth of $\mu_{\|}$at a rate that is smaller when the injection scale is larger. It is thus of interest to examine the transition from parallel heating to cooling at the level of the individual terms contributing to the r.h.s. of Eq. (2), when gradually increasing the ratio $d_{\mathrm{i}} / l_{\mathrm{f}}$ and/or $\beta$. In Fig. 5, the contributions (subject to a suitable running average in order to suppress fast oscillations) of the FLRs (red), the Hall term (green) and the heat fluxes (blue) are plotted for $\beta=0.3$ and the same three different ratios $d_{\mathrm{i}} / l_{\mathrm{f}}=0.020,0.080$ and 0.16, as in Fig. 2 . While at the largest injection scale (left panel) the heat flux (which is positive) is larger than the two other contributions, in the middle and right panels it has almost the same magnitude as the Hall effect (which is negative), so as to compensate it almost exactly. This compensation appears to be quite generic in regimes of intermediate $d_{\mathrm{i}} / l_{\mathrm{f}}$ and $\beta$. Thus, the transition from heating at large injection scales to cooling at small injection scales is governed by the FLRs, despite their relative subdominance. The increased perpendicular heating associated with this transition can be understood by noticing that the Hall term and the FLRs that contribute to $\mu_{\perp}$ act with an opposite sign in the equation for $\mu_{\|}$while, when not subdominant, the extra FLR term $\operatorname{tr}\left(\Pi_{i} \cdot \nabla u_{\mathrm{i}}\right)^{s}$ (which is positive) leads to perpendicular heating.

A similar behavior is observed when, for intermediate injection scales (e.g., $d_{\mathrm{i}} / l_{\mathrm{f}}=0.080$ ), the value of $\beta$ is increased from $\beta<1$ to $\beta>1$. This transition is exemplified in Fig. 6 for $\beta=0.1$ (bottom) and $\beta=2.4$ (top). The small $\beta$ regime is qualitatively similar to a large-scale injection case (parallel heating), while at high $\beta$, the FLRs are dominant (and negative) in the $\mu_{\|}$equation as in the small-scale injection case. On top of that, the Hall term tends to cool for $\beta<1$ and to heat for $\beta>1$ (a somewhat general property at any scale).

\subsection{The various FLR contributions}

We observed that the work of the non-gyrotropic pressure force (referred to as FLRs) plays a main role in the above transition. For example in the conditions of Fig. 5, the transition from the middle to the right panel can be sketched as follows. For the parallel quantities, the middle panel corresponds to a positive contribution of $\left(\Pi_{\mathrm{i}} \cdot \nabla u_{\mathrm{i}}\right)^{s}: \boldsymbol{\tau}$ and a negligible $\Pi_{\mathrm{i}}: \frac{d \tau}{d t}$, while in the right panel, $\Pi_{\mathrm{i}}: \frac{d \tau}{d t}$ becomes strongly negative and $\left(\Pi_{\mathrm{i}} \cdot \nabla u_{\mathrm{i}}\right)^{s}: \boldsymbol{\tau}$ subdominant. In general, in the B region of Fig. $1, \Pi_{\mathrm{i}}: \frac{d \tau}{d t}$ is dominant and negative, while in the A region, $\left(\boldsymbol{\Pi}_{\mathrm{i}} \cdot \nabla u_{\mathrm{i}}\right)^{s}: \boldsymbol{\tau}$ is dominant and positive. For the perpendicular quantities, decreasing the injection scales enhances the importance of the term $\operatorname{tr}\left(\Pi_{\mathrm{i}} \cdot \nabla u_{\mathrm{i}}\right)^{s}$, thus promoting strong heating, while in the A region, this term is almost compensated by $\left(\boldsymbol{\Pi}_{\mathrm{i}} \cdot \nabla u_{\mathrm{i}}\right)^{s}: \boldsymbol{\tau}$, making the heating very mild.

The role of the FLRs is central to triggering the emergence of mirror instability in the B region, and a sophisticated modelization of these quantities at small scales, as performed in the present FLR-Landau fluid model, turns out to be crucial to achieve such a result. It is in particular not captured when using classical large-scale FLR modeling (not shown). 

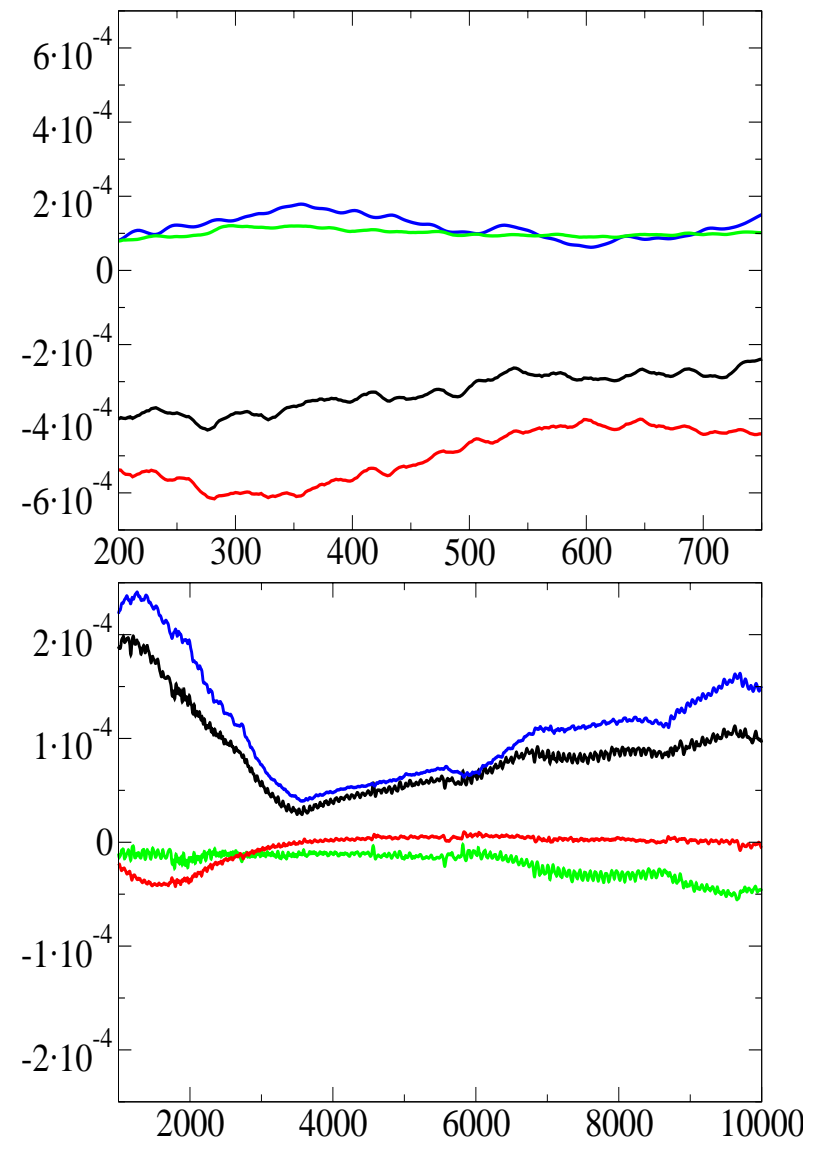

Fig. 6. Time evolution of the terms entering the r.h.s. of the equation for $\mu_{\|}$, for two runs of Fig. 3, with $d_{\mathrm{i}} / l_{\mathrm{f}}=0.080$ and $\beta=2.4$ (top) and $\beta=0.1$ (bottom). Same color code as in Fig. 5, i.e., black: total r.h.s.; blue: gyrotropic heat fluxes; green: Hall effect; red: nongyrotropic pressure.

When driving the system at scales of the order of the ion Larmor radius where stochastic heating is supposed to be most efficient, the FLRs account for most of the perpendicular heating and parallel cooling of the plasma. To study this regime, we performed several series of simulations in which we kept constant the ratio $r_{\mathrm{L}} / l_{\mathrm{f}}$ by varying simultaneously $d_{\mathrm{i}} / l_{\mathrm{f}}$ and $\beta$. The dynamics turns out to be simpler when the driving scales approach the Larmor radius, e.g., for $r_{\mathrm{L}} / l_{\mathrm{f}}=0.35$ (red series in Fig. 1). In this case, we plot in Figs. 7 and 8 for two values of $\beta$ the time variation of the r.h.s. (black lines) of Eqs. (1) and (2) for $\mu_{\perp}$ (top) and $\mu_{\|}$(bottom) respectively, together with that of both the total FLRs (orange) and their individual contributions involving $\operatorname{tr}\left(\boldsymbol{\Pi}_{\mathrm{i}} \cdot \nabla u_{\mathrm{i}}\right)^{s}(\mathrm{red}),\left(\boldsymbol{\Pi}_{\mathrm{i}} \cdot \nabla u_{\mathrm{i}}\right)^{s}: \boldsymbol{\tau}$ (green) and $\boldsymbol{\Pi}_{\mathrm{i}}: \frac{d \boldsymbol{\tau}}{d t}$ (blue). For the sake of clarity, a running average is performed on 50 time units. At small $\beta$ (e.g., $\beta=0.075$, Fig. 7), the term in $\operatorname{tr}\left(\Pi_{\mathrm{i}} \cdot \nabla u_{\mathrm{i}}\right)^{s}$ that arises only in the perpendicular equation is dominant and prescribes the growth of $\mu_{\perp}$. The main contribution to this term originates from $\Pi_{\mathrm{yz}} \partial_{\mathrm{z}} u_{\mathrm{y}}$, which involves the forced velocity component. This perpendicular ion heat-
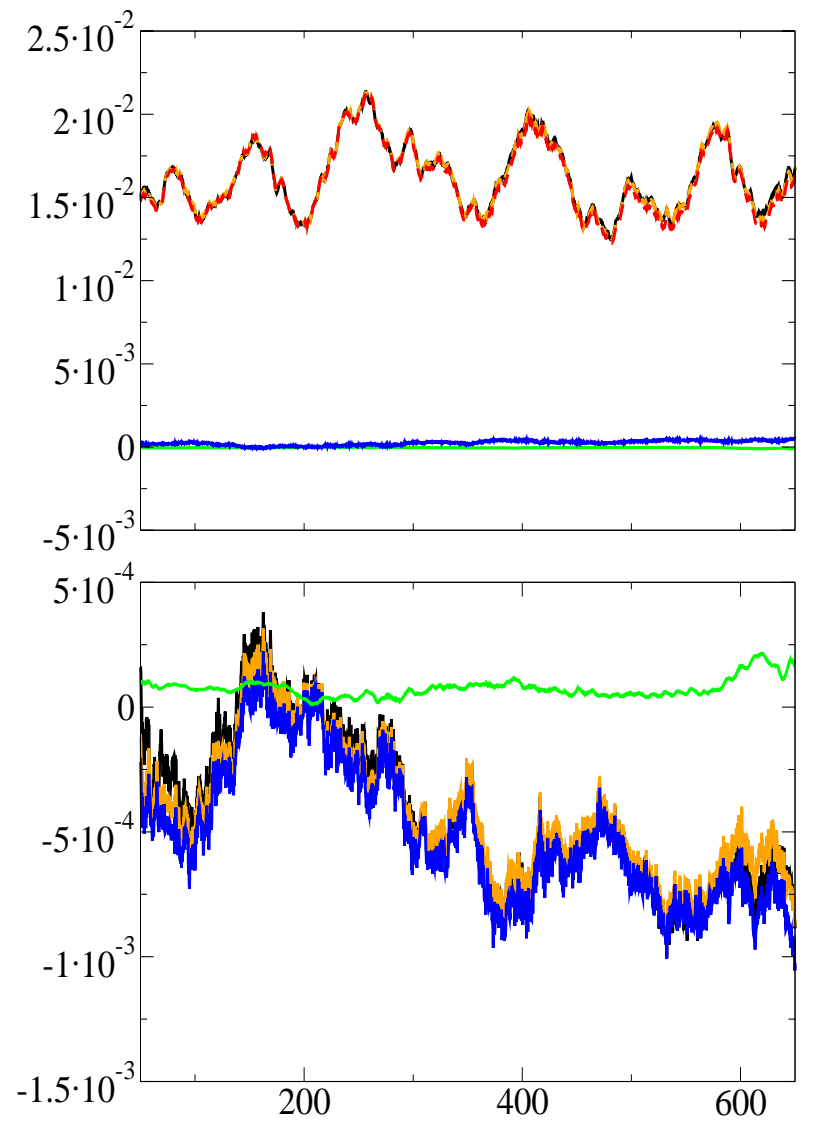

Fig. 7. Time evolution of the total r.h.s. and of the detailed contributions to the non-gyrotropic pressure in the equation for $\mu_{\perp}$ (top) and $\mu_{\|}$(bottom) for one of the runs presented in the top panel of Fig. 4 , i.e., $\beta=0.075$ and $d_{\mathrm{i}} / l_{\mathrm{f}}=1.27$. Black: total r.h.s.; orange: sum of all the non-gyrotropic pressure contributions; red: term involving $\operatorname{tr}\left(\boldsymbol{\Pi}_{\mathrm{i}} \cdot \nabla u_{\mathrm{i}}\right)^{s}$; green: term involving $\left(\boldsymbol{\Pi}_{\mathrm{i}} \cdot \nabla u_{\mathrm{i}}\right)^{s}: \boldsymbol{\tau}$; blue: term involving $\Pi_{\mathrm{i}}: \frac{d \boldsymbol{\tau}}{d t}$.

ing is in particular consistent with the small-beta stochastic heating simulations of Chandran et al. (2010). Moreover, the term in $\Pi_{\mathrm{i}}: \frac{d \tau}{d t}$ (which almost coincides with the contribution $\left.\Pi_{\mathrm{yz}} \widehat{b}_{\mathrm{z}} \frac{d \widehat{b}_{\mathrm{y}}}{d t}\right)$ accounts for essentially all the variations of $\mu_{\|}$. In this small-beta regime, we observe that $\left|u_{\mathrm{y}}\right| \gg\left|b_{\mathrm{y}}\right|$.

At larger $\beta$ (e.g., $\beta=2.4$, Fig. 8), the FLRs contribute to most of the variations of $\mu_{\perp}$ and $\mu_{\|}$, but the perpendicular heating now results from the two terms mentioned above, which contribute by a comparable amount. A small but measurable contribution to the heating also originates from the Hall term. In this regime, the magnitude of $u_{\mathrm{y}}$ and $b_{\mathrm{y}}$ are comparable. A similar pattern appears when the ratio $r_{\mathrm{L}} / l_{\mathrm{f}}$ is decreased as in the orange $\left(r_{\mathrm{L}} / l_{\mathrm{f}}=0.087\right)$ and violet $\left(r_{\mathrm{L}} / l_{\mathrm{f}}=0.062\right)$ series of Fig. 1, with an increasing importance of the Hall term and heat flux contributions. 

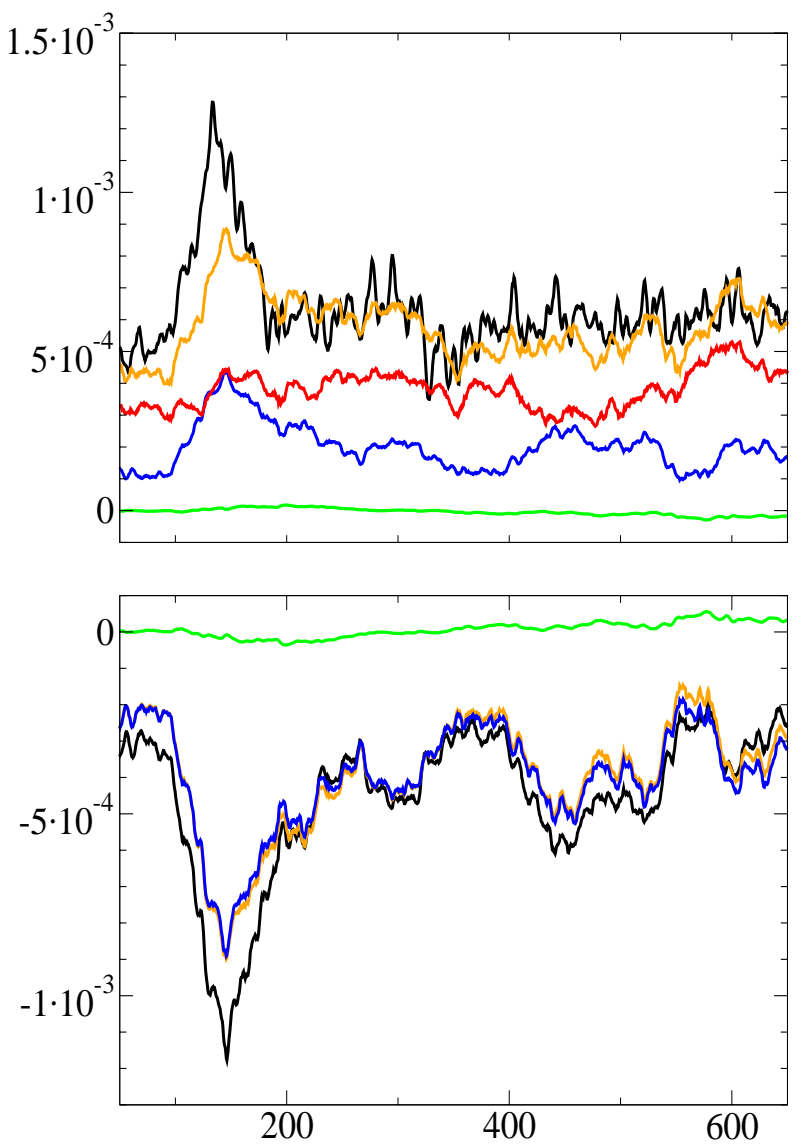

Fig. 8. Same as Fig. 7, but for another run of the top panel of Fig. 4, i.e., $\beta=2.4$ and $d_{\mathrm{i}} / l_{\mathrm{f}}=0.23$. The same color code is used: black: total r.h.s.; orange: sum of all the non-gyrotropic pressure contributions; red: term involving $\operatorname{tr}\left(\Pi_{\mathrm{i}} \cdot \nabla u_{\mathrm{i}}\right)^{s}$; green: term involving $\left(\boldsymbol{\Pi}_{\mathrm{i}} \cdot \nabla u_{\mathrm{i}}\right)^{s}: \boldsymbol{\tau}$; blue: term involving $\boldsymbol{\Pi}_{\mathrm{i}}: \frac{d \boldsymbol{\tau}}{d t}$.

\section{Dominant modes and electron heating}

In order to relate the thermal dynamics of the plasma to its global properties and in particular to the dominant type of waves that are present, it is of interest to consider the evolution of quantities such as the magnetic compressibility $\chi=\left\langle\widetilde{b}_{\mathrm{z}}^{2}\right\rangle /\left\langle\widetilde{b}_{\mathrm{z}}^{2}+b_{\perp}^{2}\right\rangle$ (where a tilde indicates fluctuations about the mean value) and the correlation between the fluctuations of the density and of the square magnetic field amplitude $\phi=\left\langle\widetilde{\rho}\left(\widetilde{b}_{\mathrm{z}}^{2}+b_{\mathrm{y}}^{2}\right)\right\rangle / \sqrt{\left\langle\widetilde{\rho}^{2}\right\rangle\left\langle\left(\widetilde{b}_{\mathrm{z}}^{2}+b_{\mathrm{y}}^{2}\right)^{2}\right\rangle}$, when varying the parameters.

In general, $\chi$ increases from the $\mathrm{A}$ to the $\mathrm{B}$ region of the perpendicular plane, indicating the presence in the latter region of a significant amount of mirror modes even before they become unstable. It in particular rapidly increases when, in the conditions of Fig. 2 (right), the perpendicular heating and parallel cooling undergo a strong enhancement at the moment when the mirror instability develops. This strengthening is related to an increase of the FLRs and in particular of
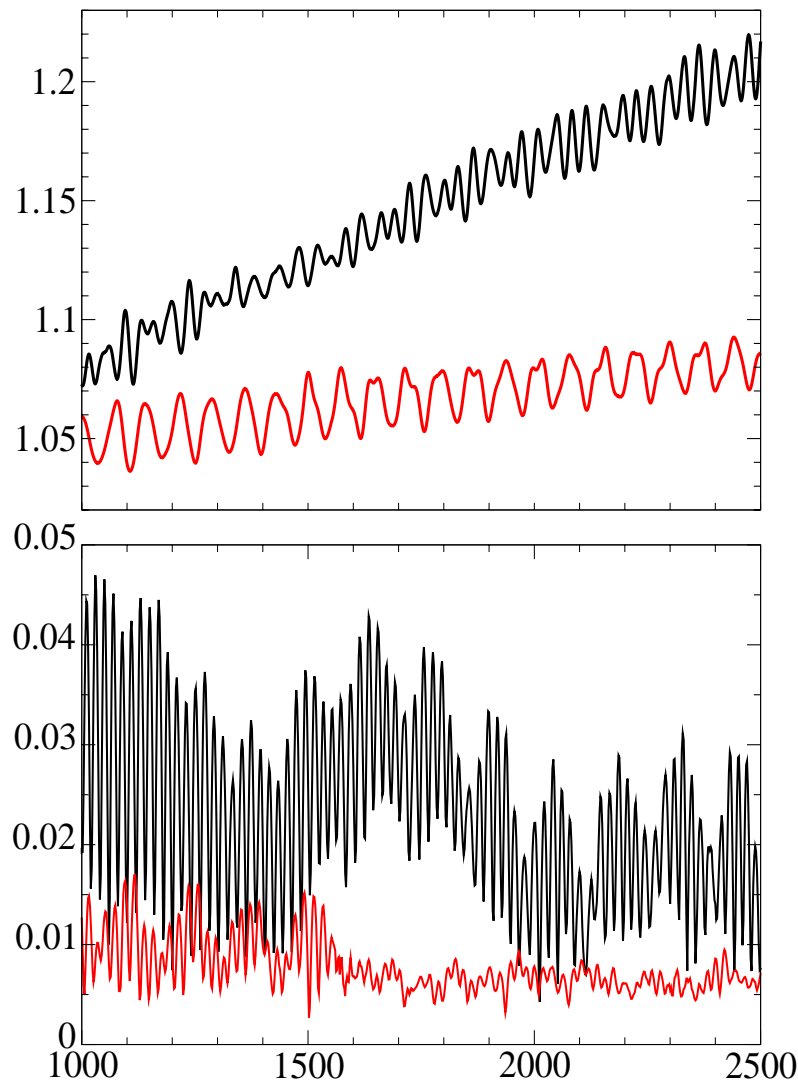

Fig. 9. Time evolution of the electron parallel temperature (top) for two runs (red or black lines) with the same parameters $\beta=0.4$, and $d_{\mathrm{i}} / l_{\mathrm{f}}=0.080$ (black point in the transition zone between the A and $\mathrm{B}$ regions in Fig. 1) for which the quantity $\sqrt{\left\langle u_{\mathrm{x}}^{2}\right\rangle}$ (bottom), which measures the level of fast magnetosonic waves, has a significantly different magnitude.

the element $\Pi_{\mathrm{yz}}$ that includes a term proportional to the temperature anisotropy (Passot and Sulem, 2007; Passot et al., 2012). Simulations performed with initially anisotropic ion temperatures $\left(T_{\perp}>T_{\|}\right)$indeed show an enhancement of the perpendicular heating (not shown).

For what concerns the correlation $\phi$, it is negative in all the simulations, indicating that the magnetosonic modes are preferentially slow, except when the injection takes place at very large scales $\left(\beta=0.3, d_{\mathrm{i}} / l_{\mathrm{f}}=0.020\right)$ for which $\phi$ is positive, indicating the dominance of fast magnetosonic waves in this case.

The amount of energy associated with fast magnetosonic modes can be estimated by the quantity $\sqrt{\left\langle u_{\mathrm{x}}^{2}\right\rangle}$ (where $x$ is the direction perpendicular to the ambient field in the plane that also includes the direction of propagation). In all the performed simulations, no clear correlation is observed between the magnitude of $\sqrt{\left\langle u_{\mathrm{x}}^{2}\right\rangle}$ and the characteristics of the ion heating. Differently, this quantity has a direct relation with the parallel electron heating (in the perpendicular direction, the electron heating is negligible). To analyze this effect, it is 
convenient to consider two runs with identical initial parameters $\beta=0.4$ and $d_{\mathrm{i}} / l_{\mathrm{f}}=0.080$ (black point in the transition zone between the regions A and B of Fig. 1), for which driving by different realizations of the same random forcing can lead to the development of different levels of $\sqrt{\left\langle u_{\mathrm{x}}^{2}\right\rangle}$ (Fig. 9, bottom) and different growth rates of the (space-averaged) parallel electron temperature (Fig. 9, top). The larger $\sqrt{\left\langle u_{\mathrm{x}}^{2}\right\rangle}$ is, the faster the temperature growth is.

Furthermore, the electron parallel heating turns out to be strongly enhanced when at fixed $\beta$ the ratio $d_{\mathrm{i}} / l_{\mathrm{f}}$ is increased. As a typical example, for $\beta=0.3$ we obtained a temperature growth rate that is almost 0 for $d_{\mathrm{i}} / l_{\mathrm{f}}=0.020$, while it is $1 \times$ $10^{-4}$ for $d_{\mathrm{i}} / l_{\mathrm{f}}=0.080,3 \times 10^{-4}$ for $d_{\mathrm{i}} / l_{\mathrm{f}}=0.11$ and $5 \times$ $10^{-4}$ for $d_{\mathrm{i}} / l_{\mathrm{f}}=0.16$. When prescribing the scale separation $d_{\mathrm{i}} / l_{\mathrm{f}}=0.080$ and varying $\beta$, the growth rate is mostly the same (about $1 \times 10^{-4}$ ) for $\beta=0.1$ and 0.3 , and decreases to about $3 \times 10^{-5}$ for $\beta=0.6,1.2$ and 2.4. This drastic change of behavior appears to be related to the transition between the $\mathrm{A}$ and $\mathrm{B}$ regions.

\section{Conclusions}

We show in this paper that the FLR-Landau fluid model allows for a self-consistent study of the non-resonant plasma heating due to random Alfvén waves. A random driving that mimics the energy injection by a turbulent cascade leads to a perpendicular heating of the ions. Furthermore, the parallel heating observed when the plasma is driven at large scale is replaced by a parallel cooling when the driving acts at scales comparable to the ion Larmor radius. Differently, the electrons are always heated in the parallel direction by Landau damping, through the gyrotropic heat fluxes at a rate that rapidly increases with the level of fast magnetosonic waves. An advantage of the present approach is the possibility to discriminate between the various contributions and to identify the dominant effects. The main role of the non-gyrotropic pressure on the ion thermal dynamics is in particular pointed out.

The present study was performed in a one-dimensional setting, along a direction quasi-transverse to the ambient magnetic field. We concentrated on heating processes due to KAWs, whose efficiency is related to the proximity of the wavelengths to the ion Larmor radius. Other heating processes, in addition to Landau and cyclotron resonances (Marsch and Bourouaine, 2011), are based on the effect of rapidly evolving small-scale structures such as currents sheets (Markovskii and Vasquez, 2011; Servidio et al., 2012). Forthcoming developments will concern the extension of the present simulations to the fully three-dimensional FLRLandau fluid model, which permits the development of turbulent cascades and thus provides a suitable tool to address the heating problem in a fully turbulent regime.
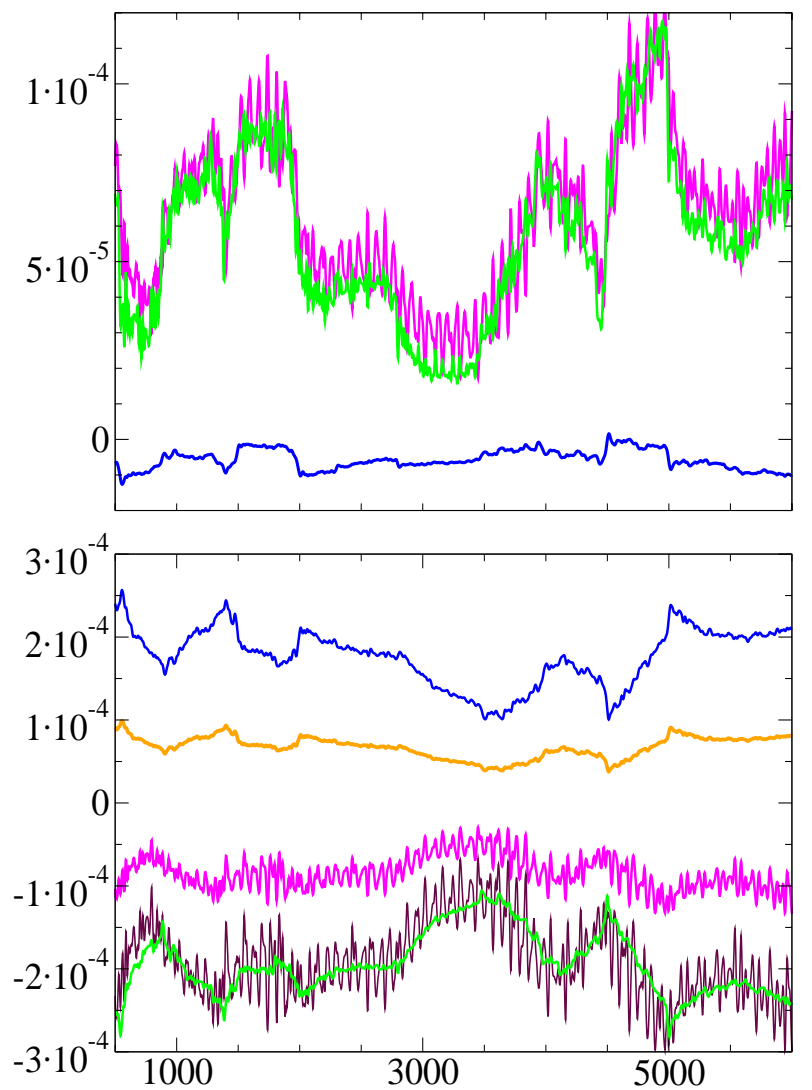

Fig. A1. For the same run as in Fig. 5 (middle) (i.e., $\beta=0.3$ and $\left.d_{\mathrm{i}} / l_{\mathrm{f}}=0.080\right)$, time evolution of the terms entering the r.h.s. of the equation for $\mu_{\perp}$ and $\bar{T}_{\perp}$ (top) and $\mu_{\|}$and $\bar{T}_{\|}$(bottom). The color code is blue for gyrotropic heat fluxes, and green for Hall effect in the $\mu$ equations; magenta for mechanical contributions, and orange for gyrotropic heat fluxes in the temperature equation. In the bottom panel, the maroon curve is the same as the magenta one after rescaling by a factor 2.25 .

\section{Appendix A}

In order to interpret the similarity in the behaviors of the temperatures and of the $\mu$ 's, announced in Sect. 2, we plot in Fig. A1 the time evolution of the different terms appearing in the right hand sides of Eqs. (1)-(4) for the same run as in Fig. 2 (middle) for which the Hall contribution is relevant. In general, all these terms have large oscillations that must be averaged out in order to extract their secular effects that are the only interesting issue for long-time plasma heating. For this purpose, a running average over 500 time units was applied to all the quantities. In the top panel that refers to the perpendicular quantities, the magenta curve corresponds to the mechanical term in the equation for $\bar{T}_{\perp \mathrm{i}}$ and the green one to the Hall term in the equation for $\mu_{\perp}$. These curves follow each other quite accurately, indicating that the Hall effect explicitly present in the $\mu_{\perp}$ equation has a counterpart 
in the secular evolution of the mechanical exchanges for $\bar{T}_{\perp \mathrm{i}}$. The blue curve represents the gyrotropic flux term in the $\bar{T}_{\perp \mathrm{i}}$ representation, which turns out to be subdominant and is not present in the equation for $\mu_{\perp}$. The non-gyrotropic pressure contributions are almost identical, and the non-gyrotropic fluxes turn out to be subdominant (not shown).

The situation is somewhat more complicated for parallel quantities (bottom panel). Keeping the same color code as above, the magenta line refers to the sum of the mechanical terms in the equation for $\bar{T}_{\| \mathrm{i}}$, and the green one to the Hall term in the equation for $\mu_{\|}$. It turns out that these quantities match each other only after rescaling the former by a factor empirically evaluated to 2.25 (maroon curve). The same ratio appears when comparing the contributions of the gyrotropic fluxes in both representations (orange and blue curves). Despite the presence of this factor, the global evolutions of $\bar{T}_{\| \mathrm{i}}$ and $\mu_{\|}$are essentially identical because, for the parallel quantities, depending on the representation, the mechanical or Hall contributions are just the opposite of the heat fluxes, so they cancel out, causing the heating properties to be governed by subdominant terms. The similar behavior of $\bar{T}_{\| \mathrm{i}}$ and $\mu_{\|}$thus relies on this approximate balance.

Acknowledgements. The research leading to these results has received funding from the European Commission's Seventh Framework Programme (FP7/2007-2013) under the grant agreement SHOCK (project number 284515). The support of INSU-CNRS "Programme Soleil-Terre" is also acknowledged.

Guest Editor M. Balikhin thanks O. A. Pokhotelov, M. Leubner, and one anonymous referee for their help in evaluating this paper.

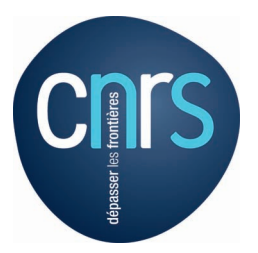

The publication of this article is financed by CNRS-INSU.

\section{References}

Borgogno, D., Passot, T., and Sulem, P. L.: Magnetic holes in plasmas close to the mirror instability, Nonlin. Processes Geophys., 14, 373-383, doi:10.5194/npg-14-373-2007, 2007.

Bourouaine, S., Marsch, E., and Vocks, C.: On the efficiency of nonresonant heating by coronal Alfvén waves, Astrophys. J., 684, L119-L122, 2008.

Camporeale, E., Passot, T., and Burgess, D.: Implications of a nonmodal theory for the marginal stability state and the dissipation of fluctuations in the solar wind, Astrophys. J., 715, 260-270, 2010.

Chandran, B. D. G., Li, B., Rogers, B. N., Quataert, E., and Germaschewski, K.: Perpendicular ion heating by low-frequency Alfvén wave turbulence in the solar wind, Astrophys. J., 720, 503-515, 2010.
Chew, C. F., Goldberger, M. L., and Low, F. E.: The Boltzmann equation and the one-fluid hydromagnetic equation in the absence of particle collisions, Proc. Roy. Soc. (London), A236, 112-118, 1956.

Hellinger, P., Trávníček, P. M., Štverák, S., Matteini, L., and Velli, M.: Proton thermal energetics in the solar wind: Helios reloaded, J. Geophys. Res., 118, 1351-1365, doi:10.1002/jgra.50107, 2013.

Hunana, P., Laveder, D., Passot, T., Sulem, P., and Borgogno, D.: Reduction of compressibility and parallel transfer by Landau damping in turbulent magnetized plasmas, Astrophys. J., 743, 128, 2011.

Hunana, P., Goldstein, M. L., Passot, T., Sulem, P. L., Laveder, D., and Zank, G. P.: Polarization and compressibility of oblique kinetic Alfvén waves, Astrophys. J., 766, 93, 2013.

Karimabadi, H., Roytershteyn, V., Wan, M., Matthaeus, W. H., Daughton, W., Wu, P., Shay, M., Loring, B., Borovsky, J., Leonardis, E., Chapman, S. C., and Nakamura, T. K. M.: Coherent structures, intermittent turbulence, and dissipation in hightemperature plasmas, Phys. Plasmas, 20, 012303, , 2013.

Laveder, D., Marradi, L., Passot, T., and Sulem, P. L.: Fluid simulations of mirror constraints on proton temperature anisotropy in solar wind turbulence, Geophys. Res. Lett., 38, L17108, doi:10.1029/2011GL048874, 2011.

Lu, Q. and Chen, L.: Ion heating by a spectrum of obliquely propagating low-frequency Alfvén waves, Astrophys. J., 704, 743749, 2010.

Markovskii, S. A. and Vasquez, B. J.: A short-timescale channel of dissipation of the strong solar wind turbulence, Astrophys. J., 739, 22, 2011.

Marsch, E. and Bourouaine, S.: Velocity-space diffusion of solar wind protons in oblique waves and weak turbulence, Ann. Geophys., 29, 2089-2099, doi:10.5194/angeo-29-2089-2011, 2011.

Nariyuki, Y., Hada, T., and Tsubouchi, K.: Heating and acceleration of ions in nonresonant Alfvénic turbulence, Phys. Plasmas, 17, 072301, doi:10.1063/1.4773205, 2010.

Passot, T. and Sulem, P. L.: Collisionless magnetohydrodynamics with gyrokinetic effects, Phys. Plasmas, 14, 082502, doi:10.1063/1.2751601, 2007.

Passot, T., Sulem, P. L., and Hunana, P.: Extending magnetohydrodynamics to the slow dynamics of collisionless plasmas, Phys. Plasmas, 19, 082113, doi:10.1063/1.4746092, 2012.

Roberts, O. W., Li, X., and Li, B.: Kinetic plasma turbulence in the fast solar wind measured by Cluster, Astrophys. J., 769, doi:10.1088/0004-637X/769/1/58, 2013.

Sahraoui, F., Goldstein, M. L., Belmont, G., Canu, P., and Rezeau, L.: Three Dimensional anisotropic k spectra of turbulence at subproton scales in the solarWind, Phys. Rev. Lett., 105, 131101, doi:10.1103/PhysRevLett.105.131101, 2010.

Servidio, S., Valentini, F., Califano, F., and Veltri, P.: Local kinetic effects in two-dimensional plasma turbulence, Phys. Rev. Lett., 108, 045001, doi:10.1103/PhysRevLett.108.045001, 2012.

Wang, C. B., Wu, C. S., and Yoon, P. H.: Heating of ions by Alfvén waves via nonresonant interactions, Phys. Rev. Lett., 96, 125001, doi:10.1103/PhysRevLett.96.125001, 2006.

Wu, C. S. and Yoon, P. H.: Proton heating via nonresonant scattering off intrinsic Alfénic turbulence, Phys. Rev. Lett., 99, 075001, doi:10.1103/PhysRevLett.99.075001, 2007. 